\title{
La paroxetina fue efectiva para el tratamiento de síncope vasovagal refractario
}

Effects of paroxetine hydrochloride, a selective serotonin reuptake inhibitor, on refractory vasovagal syncope: a randomized, double blind, placebo controlled study. Di Girolamo L, Di Iorio C, Sabatini P et al. J Am Coll Cardiol 1999;33:1227-30

\section{Objetivo}

Evaluar la efectividad de la paroxetina, un inhibidor de la recaptación de serotonina, para prevenir el síncope vasovagal en pacientes refractarios o quye no toleraron otras medicaciones.

\section{Diseño}

Ensayo clínico controlado aleatorizado, doble ciego.

\section{Lugar}

Instituto Clínico Cardiovascular de la Universidad G.D’Annunzio, Italia.

\section{Pacientes}

Se incluyeron pacientes con síncope vasovagal refractario con tilt test positivo. La etilolgía del síncope permanecía inexplicada a pesar del estudio intensivo de los pacientes conpruebas cardiológicas y neurológicas. Se excluyeron pacientes con depresión mayor o trastornos de pánico.

\section{Intervención}

Se aleatorizó a los pacientes a dos grupos: paroxetina $20 \mathrm{mg} /$ día o tabletas idénticas de placebo. Se repitió el tilt test al mes de iniciar tratamiento.

\section{Medición de los resultados}

Recurrencia de síncope espontáneo.

\section{Resultados}

El seguimiento medio fue de $25.4+/-7.9$ meses, con un rango de 24 a 33. De los 68 pacientes, 42 eran mujeres, y la edad promedio fue de $44.7+/-16.5$ años). Tomaron paroxetina 34 pacientes y placebo otros 34. Las características basales fueron similares en ambos grupos. Hubo un solo abandono en el grupo paroxetina y hubo buena tolerancia a la medicación. La paroxetina fue efectiva para disminuir las recurrencias (tabla). También redujo la tasa de inducción de síncope por tilt test al mes ( 38.2 vs $61.8 \%, p=0.001$ ). El número de síncopes anuales no se modificó en el grupo placebo pero se redujo en el grupo paroxetina (de $8.1+/-3.4$ basales a $5.9+/-1.7$ con tratamiento).

\section{Tabla: recurrencia a los dos años.}

\begin{tabular}{lcccc}
\hline Resultado & $\begin{array}{c}\text { Tasa en grupo } \\
\text { control } \\
(\mathrm{n}=34)\end{array}$ & $\begin{array}{c}\text { Tasa en grupo } \\
\text { paroxetina } \\
(\mathrm{n}=34)\end{array}$ & $\begin{array}{c}\text { RRA } \\
(\text { IC 95\%) \& }\end{array}$ & $\begin{array}{c}\text { NNT } \\
(\text { IC 95\%) \& }\end{array}$ \\
\hline $\begin{array}{l}\text { Recurrencia } \\
\text { de sincope a }\end{array}$ & $52.9 \%$ & $17.6 \%$ & $\begin{array}{c}35.3 \% \\
(14 \% \text { a } 56 \%)\end{array}$ & $\begin{array}{c}3 \\
\text { los a } 7)\end{array}$ \\
\hline
\end{tabular}

\& Datos calculados por EVIDENCIA, $\mathrm{p}<\mathbf{0 . 0 0 0 1}$

\section{Conclusiones}

La paroxetina redujo significativamente la tasa de recurrencias en pacientes con síncope vasovagal refractario a otros tratamientos; $y$ fue bien tolerada por los pacientes.

\section{COMENTARIO}

El síncope vasovagal, también llamado neurocardiogénico, mediado neuralmente, o simplemente desmayo común, es la causa más frecuente de síncope, principalmente en pacientes menores de 65 años sin cardiopatía subyacente. ${ }^{1}$

La mayor parte de las veces el diagnóstico se hace por las características clínicas del síncope (típico curso difásico, con una fase inicial de prodromos con síntomas autonómicos, a la que sucede la fase sincopal propiamente dicha). La recuperación es casi inmediata sin ningún tipo de secuela, aunque a veces puede haber traumatismos por la caída.

En muchos casos, el cuadro no es típico, por lo que es necesario recurrir a estudios complementarios como el tilt test para intentar reproducir la respuesta vasovagal y confirmar así la etiología.

Más allá de cómo se llegue al diagnóstico, los síncopes vasovagales no requieren tratamiento si son esporádicos y si el paciente no realiza actividades de riesgo (por ej. ser piloto de aviación, operar con máquinas). Sin embargo, hay pacientes en los que esta entidad es recurrente y altera significativamente la calidad de vida del paciente. Más allá de evitar situaciones desencadenantes, el tratamiento farmacológico de elección para el sínciope vasovagal son los beta bloqueantes a dosis habituales (atenolol 25-100 mg o propranolol 40-240 mg/día). ${ }^{2}$ En pacientes que persistan sintomáticos a pesar de los beta bloqueantes, existe buena evidencia, en parte aportada por este trabajo, que avala el uso de inhibidores de la recaptación de serotonina como la paroxetina (no hay aun ensayos controlados con la fluoxetina).

Otros productos evaluados en ensayos aleatorizados y doble ciego son la etilefrina, el enalapril y la midodrina (un agonista alfa). Un estudio multicéntrico demostró que la etilefrina, droga frecuentemente utilizada, es ineficaz. ${ }^{3}$ Un ensayo con pocos pacientes sugiere buenos resultados con el enalapril ${ }^{4}$, y otro evalua la midodrina en pocos pacientes5, con mejores resultados que el placebo. El principal problema de todos estos estudios es que fueron realizados en centros de derivación cardiológicos, por lo que desconocemos la validez externa* y su aplicabilidad en la atención primaria. Estudios con pacientes de centros de atención primaria aportarán más seguridad al médico que decida utilizar estas estrategias en el consultorio.

*Ver Glosario

\section{Dr. Federico Augustovski}

Unidad de Medicina Familiar y Preventiva. Hospital Italiano de Buenos Aires.

\section{Referencias}

1. Augustovski FA, Síncope. En PROFAM, Programa de Educación a Distancia en Medicina Familiar y Ambulatoria / Esteban Rubinstein, Dir. Buenos Aires: Hospital Italiano de Buenos Aires, DDI, c 1998. v2, p 89-120

2. Am Heart J 1995 Dec;130(6):1250-3

3. Mahanonda N, Bhuripanyo K, Kangkagate C, et al. Randomized double-blind, placebo-controlled trial of oral atenolol in patients with unexplained syncope and positive upright tilt table test results.

4. Raviele A, Brignole M, Sutton R, et al. Effect of etilefrine in preventing syncopal recurrence in patients with vasovagal syncope: a double-blind, randomized, placebo-controlled trial. The Vasovagal Syncope International Study. Circulation 1999 Mar 23;99(11):1452-7

5. Zeng C, Zhu Z, Liu G, et al. Randomized, double-blind, placebo-controlled trial of oral enalapril in patients with neurally mediated syncope. Am Heart J 1998 Nov; 136(5):852-8

6. Ward CR, Gray JC, Gilroy JJ, Kenny RA. Midodrine: a role in the management of neurocardiogenic syncope. Heart 1998 Jan;79(1):45-9 